CELL DEATH AND IMMUNITY O

\section{A lethal injection}

The stealthy assassin creeps up to the victim and quickly injects a dose of deadly poison. No, it's not a scene from a spy novel, but one of the ways in which the immune system's natural assassins - cytotoxic T lymphocytes and natural killer cells — can kill their target cells. The deadly poison in this case is a serine protease called granzyme $B$, and the killing mechanism is discussed by Christopher Froelich and colleagues in The Journal of Cell Biology.

Much is known about granzyme B - there is evidence that it kills with or without caspase activation, and that it causes mitochondrial permeabilization with or without members of the BCL-2 family. So Froelich and co-workers set out to assess the relative contributions of these pathways, to come up with a probable scheme for how granzyme B normally acts. To do this, they measured several different hallmarks of apoptosis - loss of mitochondrial membrane potential $\left(\psi_{\mathrm{m}}\right)$, DNA fragmentation and activation of pro-caspase precursors.

Most previous studies have been done on cell lysates, after granzyme $B$ has been delivered to its target. By contrast, Froelich and colleagues studied whole cells after delivery of the physiological form of granzyme B, a granzyme B-serglycin (SG) complex. Previous studies with lysates had indicated that granzyme B-treated cells rapidly acquire cleaved (and presumably active) pro-caspase-3. Mature caspase-3 and granzyme $B$ then process caspase- 7 to complete the initial phase of apoptosis. The authors therefore asked how the absence of pro-caspase- 3 might alter the pattern of apoptosis in whole cells after delivery of granzyme B-SG.

The authors compared MCF-7 cells that lacked procaspase-3 (MCF-7 ${ }_{\text {vec }}$ ) with cells that contained a stable transfectant that expressed this zymogen (MCF-7 $\left.{ }_{\text {casp- }-3}\right)$, after delivery of granzyme B-SG. They saw DNA fragmentation and a loss of $\psi_{\mathrm{m}}$ in the MCF-7 ${ }_{\text {casp-3 }}$ cells, but not in the MCF-7 ${ }_{\text {vec }}$ cells, indicating that caspase- 3 is essential for starting apoptosis. The authors also showed that the requirement for caspase- 3 was not due simply to the fact that caspase- 7 could no longer be activated in the caspase-3-deficient cells.

If caspase- 3 is the crucial initiator, what's the contribution of the BCL-2-family members? First, the pro-apoptotic members - such as BAX, BAK and the so-called 'BH3-only' protein BID — might be involved in mitochondrial permeabilization by forming large ion channels in the outer mitochondrial membrane. Froelich and colleagues looked into this by studying the sensitivity of Bax/Bak-deficient mouse embryo fibroblasts (MEFs) to granzyme B-mediated killing. The Bax/Bak-deficient MEFs showed lower levels of active caspase-3 intracellularly and lower rates of $\psi_{\mathrm{m}}$ loss than wild-type MEFs, which indicates that these proteins are required for the response to granzyme $B$. The authors also studied whether BID is cleaved in response to granzyme B. Unlike previous studies, however, they could not detect the truncated (active) form of BID when precautions were taken to minimize proteolysis during processing of cells for immunoblots.

A second role for certain members of the BCL-2 family is in preventing cell death. The eponymous BCL-2 has been shown to block granzyme B-mediated apoptosis, and the authors wondered whether it might do this by acting directly on caspase- 3 . They therefore looked at the effects of granzyme B-SG in a Jurkat cell line that overexpressed BCL-2. In contrast to wild-type cells, the Jurkat ${ }_{\mathrm{BCL}-2}$ cells showed no caspase- 3 activity, loss of $\psi_{\mathrm{m}}$ or DNA fragmentation. BCL-2 did not inhibit isolated active caspase- 3 , and processed caspase- 3 was observed by immunoblot, leading the authors to conclude that BCL-2 might suppress apoptosis by blocking the assembly of processed caspase- 3 .

On the basis of these and several other observations, Froelich and colleagues suggest that the predominant granzyme B death pathway starts with the activation of pro-caspase- 3 , and that the death signal is maximized by mitochondria through caspase-mediated engagement of the BH3-only/BAX/BAK pathway. The BH3-only protein that's cleaved by caspase-3 might be BID, but Froelich and colleagues' results indicate that there might be other participants too.

Alison Mitchell, Executive Editor

10) References and links Nature Reviews Molecular Cell Biology ORIGINAL RESEARCH PAPER Metkar, S. S. et al. Granzyme B activates procaspase-3 which signals a mitochondrial amplification loop for maximal apoptosis. J. Cell Biol. 160, 875-885 (2003)

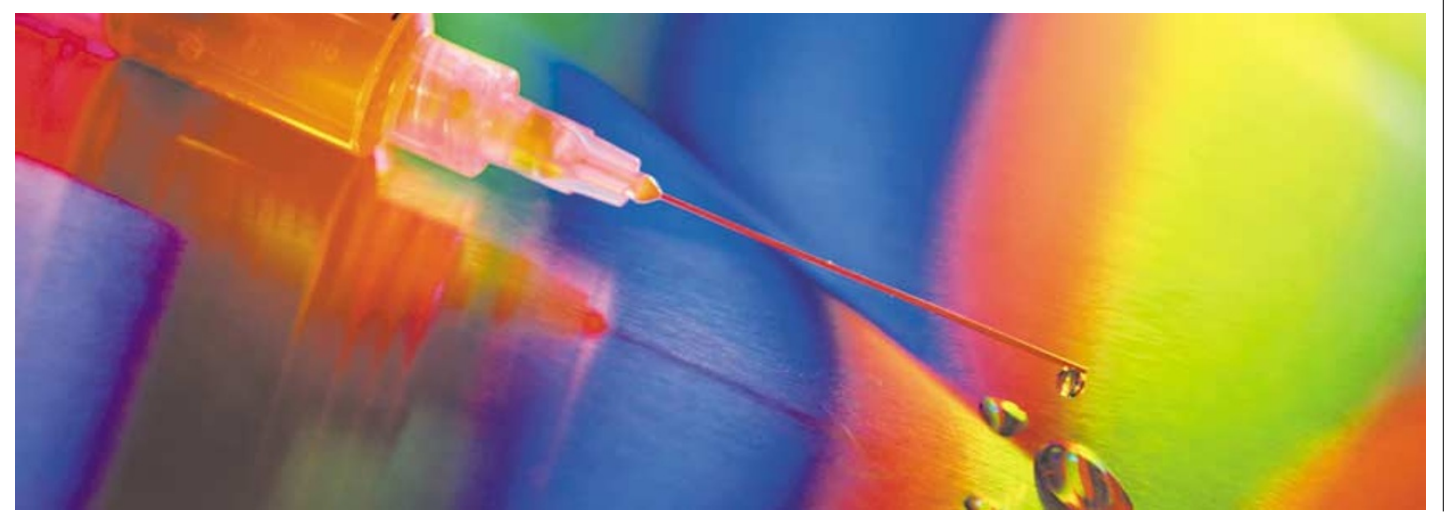

\section{IN THE NEWS}

T-cell test for TB

A new test for tuberculosis (TB) developed by Oxford Immunotec Ltd "is set to supersede the skin test", according to Ajit Lalvani (BBC News), author of a new study published in The Lancet. Peter WrightonSmith, CEO of Oxford Immunotec, said that the new test "will revolutionise TB control" by improving the detection of individuals with latent TB $(I S I S)$.

TB causes more deaths per year than any other infectious disease and the number of cases is on the increase. In Britain, the number of reported cases in 2001 was up $6 \%$ on the previous year. According to the World Health Organisation, TB is "a global health emergency", being the biggest killer of women aged 15-44 (BBC News).

Unlike the skin-prick test (given to 600,000 schoolchildren in the United Kingdom every year), which uses a crude extract of dead bacteria to induce an inflammatory reaction, the new test uses an ELISPOT technique to look for TB-specific T cells in the blood. Results are available overnight and do not require a follow-up visit. The test is less likely to give false positives than the skin-prick test, which includes proteins that are used in the BCG vaccine and is therefore confounded by TB

vaccination. The test also works well in individuals with weak immune systems, such as children, the elderly and those with AIDS. As the test can monitor how the number of specific T cells changes with time, it might also be used to monitor treatment efficacy. Although the test is more expensive than the skin prick, it should save money in the long term by eliminating the unnecessary treatment of false positives. As Lalvani noted, "The old skin test is not as cheap as it first seemed" (Reuters Health). 